\title{
Staining and High-Resolution Imaging of Three- Dimensional Organoid and Spheroid Models
}

\author{
Alejandro Lopez Gonzalez ${ }^{*}$, , Léa Luciana ${ }^{*}, 1$, Clémentine Le Nevé2 ${ }^{2}$, Julie Valantin ${ }^{2}$, Laura Francols ${ }^{2}$, Nicolas \\ Gadot $^{2}$, Christophe Vanbelle ${ }^{3}$, Laurianne Davignon ${ }^{4}$, Laura Broutier ${ }^{1}$ \\ ${ }^{1}$ Childhood Cancer \& Cell Death (C3), Université de Lyon, Université Claude Bernard Lyon 1, INSERM 1052, CNRS 5286, Centre Léon Bérard, Centre \\ de recherche en cancérologie de Lyon (CRCL), Lyon $69373^{2}$ Plateforme Anatomopathologie Recherche, Université de Lyon, Université Claude Bernard \\ Lyon 1, INSERM 1052, CNRS 5286, Centre Léon Bérard, Centre de recherche en cancérologie de Lyon (CRCL) ${ }^{3}$ Plateforme d'Imagerie cellulaire, \\ Université de Lyon, Université Claude Bernard Lyon 1, INSERM 1052, CNRS 5286, Centre Léon Bérard, Centre de recherche en cancérologie de Lyon \\ $(\mathrm{CRCL}){ }^{4}$ PerkinElmer S.A.S. \\ *These authors contributed equally
}

\section{Corresponding Author}

\section{Laura Broutier}

laura.BROUTIER@lyon.unicancer.fr

\section{Citation}

Gonzalez, A.L., Luciana, L., Le Nevé, C., Valantin, J., Francols, L., Gadot, N., Vanbelle, C., Davignon, L., Broutier, L. Staining and High-Resolution Imaging of Three-Dimensional Organoid and Spheroid Models. J. Vis. Exp. (169), e62280, doi:10.3791/62280 (2021).

\section{Date Published}

March 27, 2021

\section{DOI}

$10.3791 / 62280$

URL

jove.com/video/62280

\section{Abstract}

In vitro three-dimensional (3D) cell culture models, such as organoids and spheroids, are valuable tools for many applications including development and disease modeling, drug discovery, and regenerative medicine. To fully exploit these models, it is crucial to study them at cellular and subcellular levels. However, characterizing such in vitro $3 \mathrm{D}$ cell culture models can be technically challenging and requires specific expertise to perform effective analyses. Here, this paper provides detailed, robust, and complementary protocols to perform staining and subcellular resolution imaging of fixed in vitro 3D cell culture models ranging from $100 \mu \mathrm{m}$ to several millimeters. These protocols are applicable to a wide variety of organoids and spheroids that differ in their cell-of-origin, morphology, and culture conditions. From 3D structure harvesting to image analysis, these protocols can be completed within 4-5 days. Briefly, 3D structures are collected, fixed, and can then be processed either through paraffinembedding and histological/immunohistochemical staining, or directly immunolabeled and prepared for optical clearing and 3D reconstruction (200 $\mu \mathrm{m}$ depth) by confocal microscopy.

\section{Introduction}

Over the past decades, advances in stem cell biology and in vitro $3 \mathrm{D}$ culture technologies have heralded a revolution in biology and medicine. Higher complexity cell models in 3D have become very popular as they allow cells to grow and interact with a surrounding extracellular framework, closely recapitulating aspects of living tissues including their architecture, cell organization and interactions, or even diffusion characteristics. As such, 3D cell culture models can provide unique insights into the behavior of cells in developing or diseased tissues in vitro. Organoids and spheroids are both 
multicellular 3D structures, ranging from several micrometers to millimeters, and are the most prominent in vitro 3D structures. Both may be cultured within a supporting scaffold including (i) hydrogels derived from animals (basement membrane extract, collagen), plants (alginate/agarose), or synthesized from chemicals, or (ii) inert matrices containing pores to promote cell proliferation and growth.

Organoids and spheroids can also develop without the presence of a supporting scaffold by relying on cells to selfassemble into clusters. This relies on different techniques such as the use of non-adhesive materials to inhibit cell attachment, surface tension and gravitational force (e.g., hanging drop techniques), or constant circular rotation of vessels (e.g., spinner culture). In all cases, these techniques facilitate cell-cell and cell-matrix interactions to overcome the limitations of traditional monolayer cell culture ${ }^{1}$. The terms "organoids" and "spheroids" have been used interchangeably in the past, but there are key differences between these two 3D cell culture models. Organoids are in vitro 3D cellular clusters derived from pluripotent stem cells or tissuespecific stem cells, in which cells spontaneously self-organize into progenitors and differentiated cell types and which recapitulate at least some functions of the organ of interest ${ }^{2}$. Spheroids comprise a broader range of multicellular 3D structures formed under non-adherent conditions and can arise from a large diversity of cell types such as immortalized cell lines or primary cells ${ }^{3}$. Hence, inherent to their intrinsic stem cell origins, organoids have a higher propensity for selfassembly, viability, and stability than spheroids.
Nevertheless, in essence, these two models are 3D structures composed of multiple cells, and the techniques developed to study them are thus very similar. For example, powerful imaging approaches at the single-cell resolution level are necessary for probing the cellular complexity of both organoids and spheroids. Here, by summarizing this group's expertise and that of leaders in the field of organoids ${ }^{4}$, this paper describes detailed procedures to perform twodimensional (2D) and 3D whole-mount staining, imaging, and analyses of the cellular and subcellular composition and spatial organization of organoids and spheroids ranging from $100 \mu \mathrm{m}$ to several millimeters. Indeed, this procedure presents two different and complementary types of staining and imaging acquisition to analyze a large variety of sizes and types of in vitro 3D cell culture models. The use of one (3D whole-mount analysis) or the other (2D section analysis) will depend on the model studied and the answers sought. 3D whole-mount analysis by confocal microscopy can, for instance, be applied to visualize cells in 3D culture up to $200 \mu \mathrm{m}$ in depth, irrespective of the overall size of the $3 \mathrm{D}$ structure, whereas the analysis of $2 \mathrm{D}$ sections provides insights into samples of any size, albeit at the 2D level. This procedure has been successfully applied across a variety of organoids ${ }^{4,5}$ and spheroids derived from human and murine cells, originating from different embryonic germ layers. The overview of the procedure is shown in Figure 1. The major stages, the relationships between them, decisive steps, and the expected timing are indicated. 


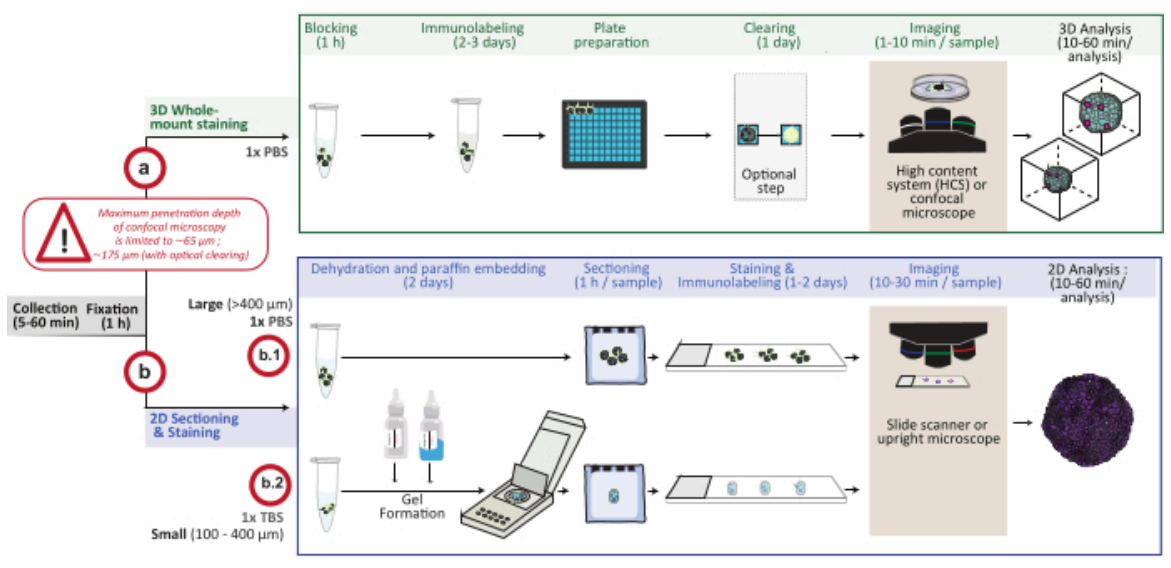

Figure 1: Schematic overview of the procedure. In vitro 3D cell culture models are collected and fixed, then either prepared for 3D whole mount staining (option a) or embedded in paraffin for 2D sectioning and staining (option b). For 3D whole-mount staining experiments, fixed 3D structures are immunolabeled following the fixation step. An optional opticalclearing step can be performed to improve imaging quality and depth of optical microscopy by reducing light scattering during image processing. Images are captured on an inverted confocal microscope or a confocal high content system and analyzed using the appropriate software. For paraffin embedding, 3D structures are directly processed (option b.1 for large structures $\geq \mathbf{4 0 0} \boldsymbol{\mu m}$ ) or included in a gel (b.2; small structures $\leq \mathbf{4 0 0} \boldsymbol{\mu m}$ ) for dehydration and paraffin embedding. Paraffin blocks are then cut and stained (histological or immunochemical staining). Images of 2D sections are obtained on a digital slide scanner or an upright microscope and analyzed on an image analysis platform using fast digital quantitative analysis. Please click here to view a larger version of this figure.

\section{Protocol}

NOTE: $A$ loss of $\leq 25 \%$ of the initial number of $3 D$ structures should be expected during the steps involving reagent changes and washing in the following procedure. Plan to use a final number of at least ten 3D structures, with a size ranging from 100 to $500 \mu \mathrm{m}$, per tested condition to perform qualitative and quantitative image analyses. If necessary, for larger structures, cut the ends of $1 \mathrm{~mL}$ pipette tips to avoid breaking the structures. For all steps, if 3D structure sedimentation is too long, cells can be gently spun at $50 \times g$ for $5 \mathrm{~min}$ at room temperature (RT). Depending on the issue investigated, advantages/disadvantages of such a spinning step should be considered, as centrifugation can compromise the shape of the 3D structures. Avoid spinning at $>100 \times g$.

\section{Collection and fixation of 3D cell culture models}

NOTE: Be careful not to aspirate the 3D structures, which will be only loosely attached to the tube wall.

1. Harvesting of $3 D$ cell culture models embedded in a matrix

NOTE: This section describes the recovery of $3 \mathrm{D}$ structures grown in drops of a basement membrane 
extract from murine Engelbreth-Holm-Swarm sarcoma (BME), but may be adapted to other matrices. See the discussion for crucial points regarding ECM.

1. Remove the culture medium from the wells without disrupting the 3D matrix. Precoat the inside and outside of a $1 \mathrm{~mL}$ pipette tip with protein (called precoated $1 \mathrm{~mL}$ tip hereafter) by dipping the full length of the tip in $0.1 \%$ bovine serum albumin (BSA) in phosphate-buffered saline (PBS) (called PBSBSA $\mathbf{0 . 1 \%}$ solution hereafter) and pipetting $1 \mathrm{~mL}$ of this solution up and down twice.

NOTE: This precoating will prevent the cells from sticking to the tip and minimize any loss.

2. Precoat the inside of a centrifuge $(15 \mathrm{~mL})$ tube with protein (called precoated centrifuge tube hereafter) by repeatedly filling with PBS-BSA $0.1 \%$ solution and emptying the tube.

NOTE: This will prevent the cells from sticking to the tube and minimize any loss.

3. Using the precoated $1 \mathrm{~mL}$ tip, carefully resuspend the 3D structures of the well using $1 \mathrm{~mL}$ of ice-cold $1 \mathrm{x}$ PBS, and gently transfer the suspension containing the $3 \mathrm{D}$ structures to the precoated centrifuge tube.

4. Gently add $13 \mathrm{~mL}$ of ice-cold $1 \times$ PBS, and allow the $3 \mathrm{D}$ structures to sediment on ice for at least $10 \mathrm{~min}$. NOTE: If required, spin for $5 \mathrm{~min}$ at $50 \times g$ at $4{ }^{\circ} \mathrm{C}$. Avoid spinning $>100 \times g$, as this will compromise the shape of the 3D structures.

5. Remove the supernatant. Using a precoated $1 \mathrm{~mL}$ tip, gently resuspend the 3D structures in $1 \mathrm{~mL}$ of icecold 1x PBS. Repeat steps 1.1.4 to 1.1 .5 to obtain a homogenous pellet without any $3 \mathrm{D}$ matrix residue.
NOTE: Efficient matrix removal is influenced by the type of matrix, the number, and size of $3 D$ structures and requires optimization for different culture conditions. For 3D structures grown in BME, recovery from the matrix removal typically takes 45-60 min.

6. Using a precoated $1 \mathrm{~mL}$ tip, transfer the $1 \mathrm{~mL} 1 \mathrm{x}$ PBS suspension containing the $3 D$ structures to a precoated $1.5 \mathrm{~mL}$ centrifuge tube, and proceed with section 1.3 .

2. Harvesting floating $3 D$ cell culture models

1. Using a precoated $1 \mathrm{~mL}$ tip, carefully collect and transfer the $3 \mathrm{D}$ structures to a precoated $1.5 \mathrm{~mL}$ centrifuge tube. Allow the 3D structures to sediment, or spin for $5 \mathrm{~min}$ at $50 \times g$ at RT.

2. Remove the supernatant. Using a precoated $1 \mathrm{~mL}$ tip, resuspend the 3D structures in $1 \mathrm{~mL}$ of $1 \times$ PBS. Proceed with section 1.3.

3. Fixation of 3D cell culture models

1. Allow the $3 D$ structures to sediment. Carefully remove the supernatant; under a fume hood, gently resuspend the $3 D$ structures in $1 \mathrm{~mL}$ of formalin using a precoated $1 \mathrm{~mL}$ tip.

NOTE: Formalin contains formaldehyde, which is hazardous. Manipulate the chemical in a chemical hood. Wear rubber gloves and safety eye goggles.

2. Incubate the $3 D$ structures for $30 \mathrm{~min}$ at $R T$.

NOTE: A 30 min fixation step with formalin is required for immunostaining of a wide range of $3 \mathrm{D}$ structures (varying in size, shape, and origin). However, in general, longer fixation times (>3 h) are better suited to preserve the fluorescence of reporter proteins. 
3. Allow the $3 D$ structures to sediment, or spin for 5 $\min$ at $50 \times g$ at RT. Gently remove the formalin, and replace it with $1 \mathrm{~mL}$ of $1 \times$ PBS. Repeat this washing step in 1x PBS twice. Store the samples at $4{ }^{\circ} \mathrm{C}$, and proceed with section 2 or section 3 .

NOTE: The protocol can be paused here, and the cells can be maintained at $4{ }^{\circ} \mathrm{C}$ for long-term storage (>1 year).

\section{3D whole mount staining, imaging, and analysis of 3D cell culture models}

NOTE: As the organoids are loosely attached to the tube wall, handle them gently as all following reagent changes can cause sample loss. Before starting, ensure the availability of the correct controls for staining. Positive and negative controls can be cells, in which the protein of interest is known to be either overexpressed or absent, respectively. Incubate samples without the primary antibody to determine if the observed signal is due to non-specific binding of the secondary antibody. As some cells tend to display high levels of autofluorescence, use controls devoid of secondary antibody to determine if the observed fluorescence is coming from background autofluorescence. Immunolabeling and fluorescent reporter visualization can be combined.

\section{1. $3 \mathrm{D}$ whole mount staining}

1. Prepare the permeabilization-blocking (PB) solution by supplementing $1 \mathrm{x}$ PBS with $0.1 \%-1 \%$ of a non-ionic surfactant (see the Table of Materials), $1 \%$ dimethylsulfoxide, $1 \%$ BSA, and $1 \%$ donkey serum (or from the animal in which the secondary antibodies were raised).

NOTE: Carefully optimize the concentration of the non-ionic surfactant depending on the localization of the target: membrane (0-0.5\%), cytoplasm $(0.5-1 \%)$, and nucleus (1\%). This solution can be stored at $4{ }^{\circ} \mathrm{C}$ for up to 1 month. BSA usually works well for the blocking step, but in case of high background noise, perform an empirical test to obtain the best possible results for a given combination of antibodies.

2. Transfer the organoids from the $1.5 \mathrm{~mL}$ centrifuge tube to a $0.5 \mathrm{~mL}$ tube using a precoated $1 \mathrm{~mL}$ tip. Let the organoids sediment, gently remove the $1 \times$ PBS, and replace it with $0.5 \mathrm{~mL}$ of PB solution. Incubate the organoids with gentle horizontal agitation (30-50 rpm) for $1 \mathrm{~h}$ at RT.

3. Let the organoids sediment, gently remove the PB solution, and wash twice in $1 \mathrm{~mL}$ PBS-BSA $0.1 \%$ for $3 \mathrm{~min}$.

NOTE: Waiting for $3 \mathrm{~min}$ allows the structures to sediment at the bottom of the tube.

4. Gently remove the PBS-BSA $0.1 \%$, and add 250 $\mu \mathrm{L}$ of primary antibody diluted at the appropriate concentration in PB:1x PBS (1:10) solution. To prepare $10 \mathrm{~mL}$ of PB:1x PBS (1:10) solution, dilute $1 \mathrm{~mL}$ of PB solution in $9 \mathrm{~mL}$ of $1 \mathrm{x}$ PBS. Incubate for 2-3 days with gentle horizontal agitation (30-50 rpm) at $4{ }^{\circ} \mathrm{C}$.

NOTE: An appropriate antibody incubation time is crucial for a suitable antibody penetration as 3D structures can sometimes reach large sizes.

5. Let the organoids sediment, and gently remove the primary antibody solution. Wash $5 x$ in PBS-BSA $0.1 \%$ for 3 min per wash and then $2 x$ in $1 \mathrm{~mL}$ PBSBSA $0.1 \%$ for 15 min per wash with gentle horizontal agitation.

6. Add $250 \mu \mathrm{L}$ of secondary antibody diluted at $1: 250$ in PB:1x PBS (1:10) solution. Incubate for $24 \mathrm{~h}$ at $4^{\circ} \mathrm{C}$ 
with gentle horizontal agitation (30-50 rpm). For this step, protect the samples from light.

7. Add $250 \mu \mathrm{L}$ of Hoechst $33342(20 \mu \mathrm{M}$ stock solution) diluted at 1:1000 in PB:1x PBS (1:10) solution, and incubate for another $2 \mathrm{~h}$ at $4{ }^{\circ} \mathrm{C}$ with gentle horizontal agitation (30-50 rpm).

8. Let the organoids sediment, and gently remove the solution containing secondary antibody + Hoechst 33342. Wash the organoids $5 \mathrm{x}$ in $1 \mathrm{~mL}$ of $1 \mathrm{x}$ PBS for 3 min per wash and then $2 x$ in $1 \mathrm{~mL}$ of $1 x$ PBS for 15 min per wash with gentle horizontal agitation (30-50 rpm).

NOTE: It is crucial to extensively wash the samples to avoid background noise or loss of signal.

9. Store the samples in PBS at $4{ }^{\circ} \mathrm{C}$ until image acquisition. Proceed with section 2.2.

NOTE: The protocol can be paused here, and the samples can be stored at $4{ }^{\circ} \mathrm{C}$ for several months, protected from light.

2. Sample preparation for confocal imaging

1. Using a precoated $1 \mathrm{~mL}$ tip, carefully transfer the organoids into $50 \mu \mathrm{L}$ of the $1 \times$ PBS per well in a 96well black polystyrene microplate. Proceed with step

\section{2 .3 or section 2.3 .}

NOTE: At this stage, the sample can be protected from light and stored at $4{ }^{\circ} \mathrm{C}$ for many weeks.

\section{Clearing}

NOTE: The clearing step is optional and can be used to either immunolabel organoids or to detect endogenous fluorescence. Clearing can cause 3D structure shrinkage, but does not change the general morphology except for spherical monolayered organoids with large lumens ${ }^{4}$. For these cystic organoids, skip the clearing step, and perform deep-tissue imaging ${ }^{6}$.

1. Prepare $2.5 \mathrm{M}$ glycerol-fructose clearing solution containing $50 \% \mathrm{v} / \mathrm{v}$ glycerol, $11 \% \mathrm{v} /$ $\mathrm{v}$ of distilled water, and $45 \% \mathrm{w} / \mathrm{v}$ fructose by mixing on a magnetic stirrer at least overnight until the solution is completely solubilized and homogenous. Store at $4{ }^{\circ} \mathrm{C}$ in the dark for up to 1 month.

2. Remove as much $1 \times$ PBS as possible without touching the organoids. Add $200 \mu \mathrm{L}$ of the clearing solution using a $1 \mathrm{~mL}$ pipette tip after removing the end, and resuspend gently to prevent the formation of bubbles. Incubate at $\mathrm{RT}$ for at least $12 \mathrm{~h}$, and proceed with section 3 . NOTE: As the clearing solution is viscous, small volumes are difficult to handle. To facilitate handling, make sure the solution is at RT, and pipette slowly. For optimal clearing, allow the sample to sediment in the clearing solution for at least $24 \mathrm{~h}$ before imaging. If 3D structures are floating at the time of acquisition, perform an optional spin for $10 \mathrm{~min}$ at $<100 \times g$ at RT, or allow more time (one to several days) to let them sediment. The protocol can be paused at this step before proceeding to imaging if it is protected from light and stored at $4{ }^{\circ} \mathrm{C}$ (for weeks) or $-20^{\circ} \mathrm{C}$ (for months).

3. Image acquisition and analysis

NOTE: Image sectioning technology will be required to image 3D structures.

1. Use confocal microscopes, and favor immersion objectives with higher numerical aperture (NA) 
compared to air. Choose magnification objectives $(10 x, 20 x, 40 x)$ according to the size of 3D structures, image reconstruction (stitching), and solutions used for the analysis.

2. When selecting the acquisition mode, take into consideration the depth of focus of the objective used to define the step for $Z$ stacking; allow for optimal 3D rendering.

NOTE: Image analysis solutions vary, and the analysis will need to be adjusted to the software used. For instance, this analysis protocol was established on a high-content analysis software (see Table of Materials and Supplementary Figure 1 for details) and provides data on object segmentation, calculation of properties, and cell population selection within a $3 \mathrm{D}$ reconstructed object.

\section{2D sectioning, staining, imaging, and analysis of 3D cell culture models}

NOTE: 3D cell culture models vary in size. Proceed with section 3.1 or 3.2 for efficient paraffin embedding (Figure 2). Allow sufficient time for 3D structure sedimentation before any washes and reagent changes. Be careful not to aspirate the organoids that will be floating at the bottom of the tube. For paraffin embedding, refer to Figure 2 for guidance.

1. Paraffin embedding of large $(\varnothing \geq 400 \mu \mathrm{m}) 3 \mathrm{D}$ cell culture models

1. On the day before embedding, prewarm two 150 $\mathrm{mL}$ flasks filled with paraffin (paraffin baths), a small metal embedding mold per sample, and fine forceps to $65^{\circ} \mathrm{C}$.
2. Using a precoated $1 \mathrm{~mL}$ tip, carefully transfer the organoids in 1x PBS to a flat-bottom glass tube with a polytetrafluoroethylene-lined bottle cap. Let the organoids sediment, carefully remove the $1 \mathrm{x}$ PBS, and replace it with $70 \%$ ethanol. Incubate for at least $30 \mathrm{~min}$.

3. Let the organoids sediment, and carefully remove the $70 \%$ ethanol. Replace it with $1 \mathrm{~mL}$ of ready-touse eosin $\mathrm{Y}$ solution. Flick the tube, and stain for at least $30 \mathrm{~min}$. Carefully remove the eosin solution, and dehydrate the organoids in three successive washes with $1 \mathrm{~mL}$ of $100 \%$ ethanol for $\sim 30$ min each. NOTE: Ethanol, a flammable and volatile liquid, causes severe eye and respiratory tract irritation. Manipulate it in a fume hood, and wear protective eye goggles.

4. Carefully remove the $100 \%$ ethanol, and under a chemical hood, clear the organoids in 3 successive washes with $1 \mathrm{~mL}$ of xylene for $\sim 30$ min each.

NOTE: Xylene is a toxic, liquid flammable whose vapors may cause irritation. Manipulate it in a fume hood. Avoid direct contact with skin, and wear rubber gloves and protective eye goggles.

5. Under a chemical hood, prepare a white microtwin tissue cassette by placing a piece of biopsy pad (previously soaked in xylene) inside one of the compartments of the cassette. Carefully transfer the 3D structures using a precoated $2 \mathrm{~mL}$ plastic Pasteur pipette to the biopsy pad. Cover them with another biopsy pad soaked in xylene to prevent the organoids from moving, and close the cassette.

6. If several samples are processed, place the cassette in a xylene bath to await further processing. Once 
all samples are transferred into cassettes, place the cassettes in a prewarmed paraffin bath for $30 \mathrm{~min}$ at $65^{\circ} \mathrm{C}$. Transfer the cassettes to a fresh prewarmed paraffin bath overnight.

7. After paraffin impregnation, take a prewarmed embedding mold, and add the heated paraffin to it. Place the biopsy pad containing the 3D structures into the mold, and gently agitate it until all of the organoids drop to the bottom of the mold. Very carefully place the $3 \mathrm{D}$ structures at the center of the mold using prewarmed fine forceps. Proceed with section 3.3.

NOTE: Be careful not to disrupt the 3D structures with the forceps; push, but do not pinch them.

2. Paraffin embedding of small $(\varnothing \leq 400 \mu \mathrm{m}) 3 \mathrm{D}$ cell culture models

1. On the day before embedding, prewarm two 150 $\mathrm{mL}$ flasks filled with paraffin (paraffin baths), a small metal embedding mold per sample, and fine forceps to $65^{\circ} \mathrm{C}$.

2. Carefully remove the $1 x$ PBS from the organoid suspension. Gently perform 3 washes in $1 \mathrm{~mL}$ of $1 \mathrm{x}$ Tris-buffered saline (TBS). Remove as much 1x TBS as possible without touching the organoids.

NOTE: Be careful not to aspirate the sample. If necessary, perform a $5 \mathrm{~min}$ spin at $50 \times \mathrm{g}$ at RT. Remaining traces of phosphate will interfere with the following steps, notably preventing gel polymerization. Therefore, do not use PBS solutions during any processing step. For this step, a commercial kit, containing cassettes, Reagent \#1 (clear fluid), and Reagent \#2 (colored fluid), was used to facilitate the paraffin-embedded procedure without potentially loosing tiny fragments (see Table of Materials). Follow kit instructions. The cassettes are preassembled with backing papers and board inserts already in place.

3. Add 2 drops of Reagent \#2 into the tube, and mix gently by tapping the tube. Add 2 drops of Reagent $\# 1$, and mix again by tapping to make the gel solidify. Using the fine forceps, remove the gel from the tube, and place it in the well of the cassette.

4. Under the fume hood, dehydrate the sample by placing the cassette in successive baths as follows (use the $150 \mathrm{~mL}$ flasks, and use fresh ethanol or xylene for each bath): ethanol 70\%, 30 min; ethanol $96 \%, 30 \mathrm{~min}$; ethanol $100 \%$, three washes, $30 \mathrm{~min}$ each; xylene, three washes, 30 min each.

5. Place the cassettes in a prewarmed paraffin bath for $30 \mathrm{~min}$ at $65{ }^{\circ} \mathrm{C}$, and transfer them to a fresh prewarmed paraffin bath overnight. After paraffin impregnation, take a prewarmed embedding mold, and add heated paraffin into it. Open the cassette, carefully dislodge the gel with fine forceps, and place the gel containing the 3D structures onto the center of the embedding mold. Proceed with section 3.3.

3. Common steps for paraffin embedding

1. Gently transfer the mold to a cold area to let the paraffin solidify in a thin layer, which will maintain the 3D structures in the appropriate position. Add a tissue cassette on top of the mold, and add hot paraffin to cover this plastic cassette. Remove the mold once it is completely solidified, and proceed with section 3.4 .

NOTE: Paraffin blocks can be stored at room temperature for years. 
A

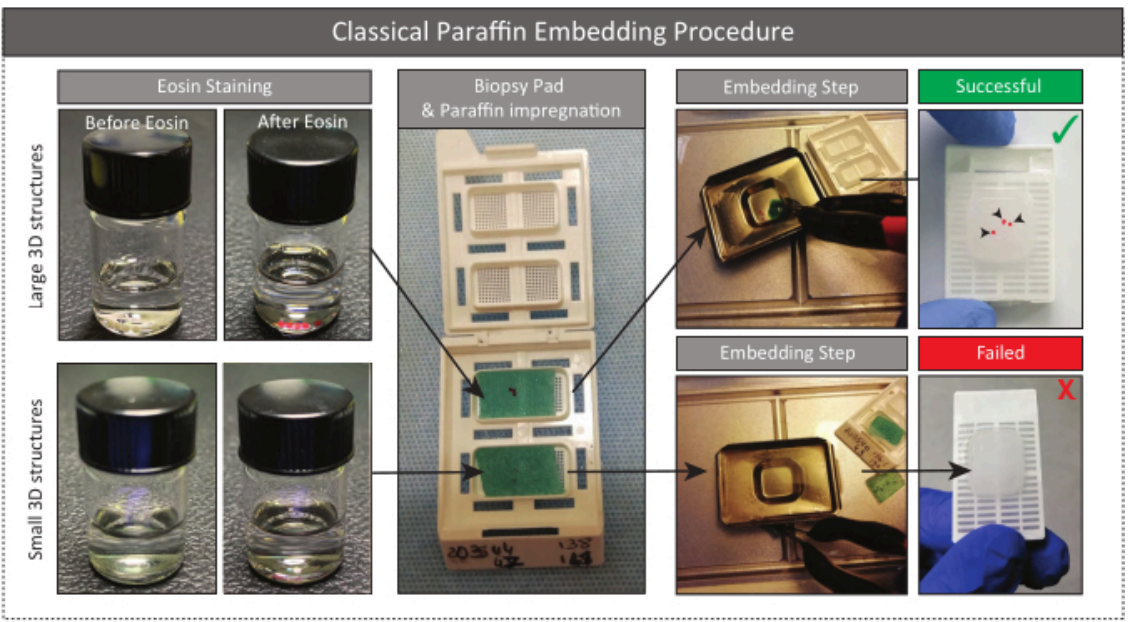

B

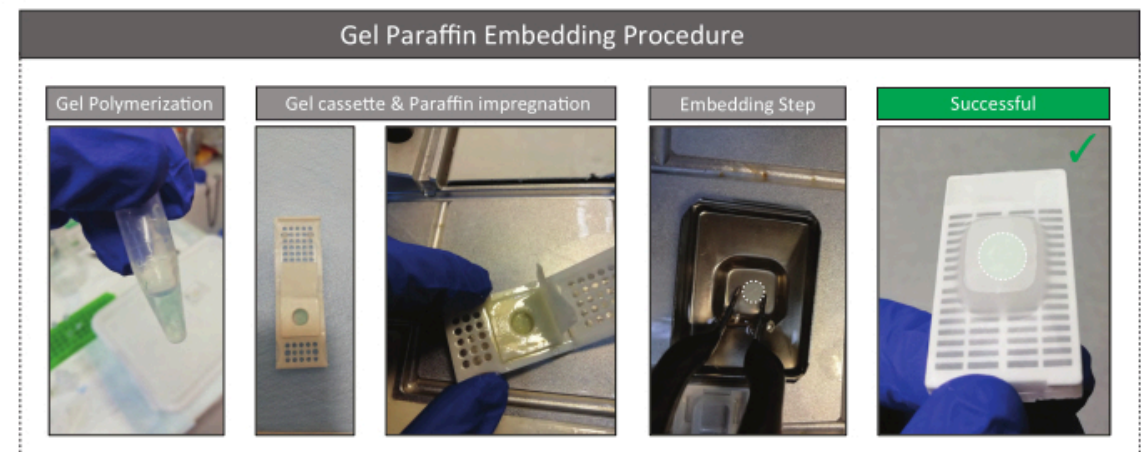

Figure 2: Overview of the procedure for paraffin embedding of large and small in vitro 3D cell culture models.

(A) Standard procedure for paraffin embedding. After fixation and dehydration, 3D structures are stained with eosin to facilitate their visualization (top and bottom left). 3D structures are carefully placed on the biopsy pad (blue) in the cassette using a $2 \mathrm{~mL}$ Pasteur pipet (middle). After paraffin impregnation, the 3D structures are gently dropped into the liquid paraffin using forceps and gently agitated in the biopsy pad. Small 3D structures are lost during this step as they cannot be released from the pad (bottom right: failed embedding). Only large 3D structures will be embedded (top right: successful embedding). Arrowheads point to 3D cultures. (B) Alternative to the standard paraffin embedding protocol. After having fixed small 3D structures, a commercial kit is used to maintain cells in a gel and facilitate their transfer to the mold after paraffin impregnation (right: successful embedding). Please click here to view a larger version of this figure.

4. Block sectioning and staining

1. Cut $4 \mu \mathrm{m}$ sections using a standard microtome, and perform standard histological and immunohistochemical techniques. Proceed with section 3.5 .

NOTE: Specific slides (see Table of Materials) were used for a better adhesion of sections. The slides 
can be stored at room temperature or at $4{ }^{\circ} \mathrm{C}$ for years.

5. Image acquisition and analysis

1. Perform imaging using a digital slide scanner or upright microscope, and analyze data using a platform for fast digital quantitative analysis that reports morphological and multiplexed expression data on a cell-by-cell basis across entire 3D structure sections (see Supplementary Figure 2 for details). NOTE: The $20 x$ objective is used routinely by this group.

\section{Representative Results}

This protocol provides an overview of the critical steps for 2D and 3D whole-mount staining, as well as imaging and quantitative analyses of 3D cell culture models (Figure 3 and

Figure 4). It is applicable to a wide range of 3D cell culture models-from spheroids to organoids from different host species or tissues-and enables the acquisition of accurate and quantitative information on architecture, cell organization, and interactions at cellular and subcellular levels (Figure
3 and Figure 4). Laboratories may need to optimize 2D histological and immunohistochemical techniques and antibody concentrations according to their own needs.

Both methods yield valuable biological information. 3D wholemount staining and confocal microscopy provide visual information on cellular composition and spatial position with a field of depth of up to $200 \mu \mathrm{m}$ (Figure 3B). However, 2D sectioning is convenient for larger $3 D$ structures to reveal detailed cellular morphological traits in the entire section of 3D structures that can be otherwise challenging to observe in situ due to light scattering that compromises resolution in larger samples. Moreover, both techniques can provide quantitative data. Indeed, the resolution obtained allows the application of cellular and subcellular segmentation algorithms for the quantification of the number of cells and the detection of the presence of various cell markers in different cellular subtypes (Figure 3F and Figure 4). In summary, the imaging techniques described here are reproducible, simple, and complementary and represent valuable tools for studying cellular heterogeneity. 

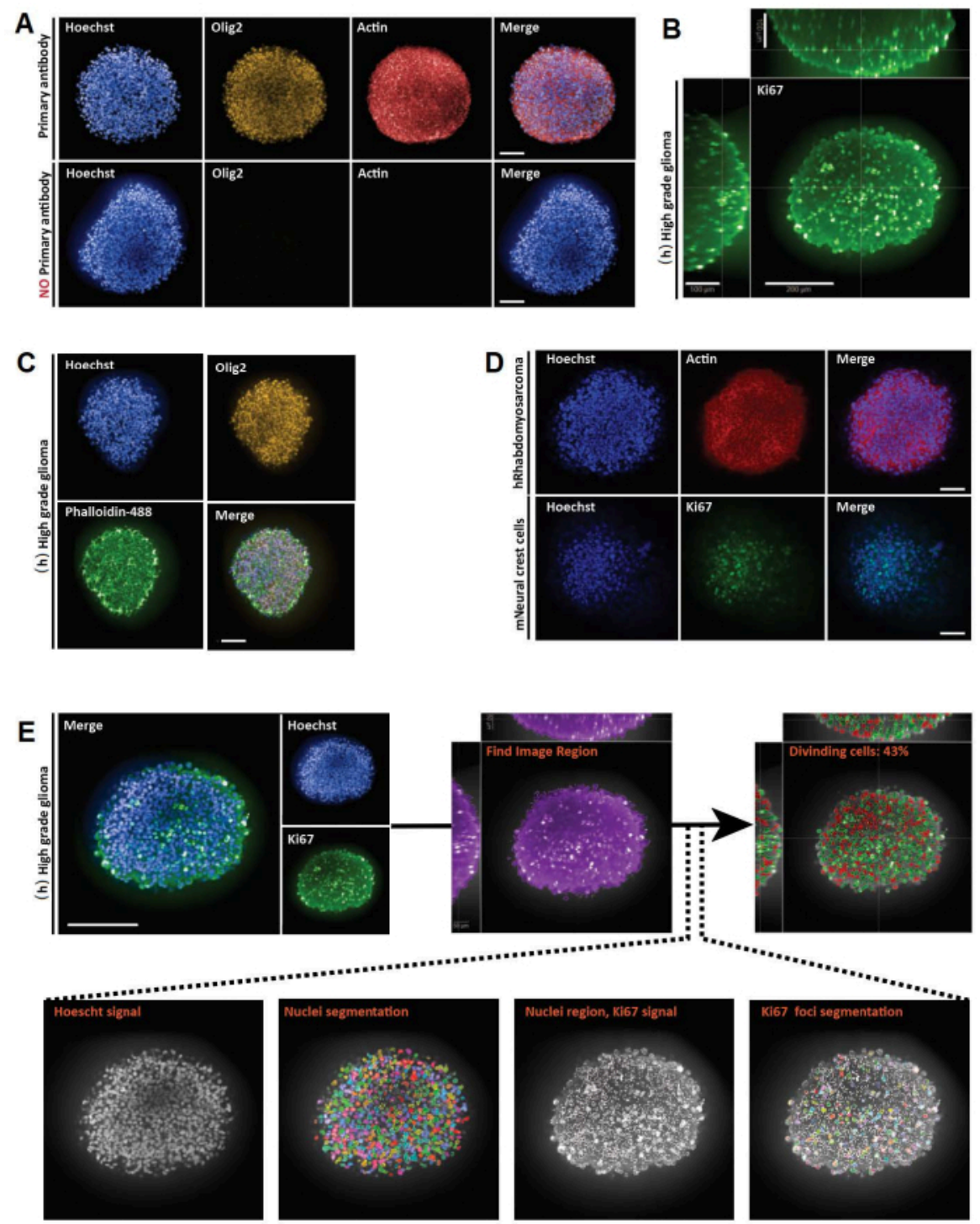

Figure 3: Representative results for 3D whole mount, imaging, and analyses of 3D and 2D optical sections.

(A) Confocal images of human (h) high-grade glioma spheroid cultured for a week and labeled with Hoechst (blue), Olig2 (yellow), and Actin (red) (20x water objective). For all acquired images, microscope settings were established using a positive control (top), and then the negative control was imaged using identical settings to control the lack of fluorescence in the absence of primary antibody (bottom). (B) Orthogonal 3D whole-mount representation of Ki67 staining performed in (h) high-grade glioma spheroid cultured for a week (glycerol-fructose clearing; 20x water objective, confocal). (C) Confocal images of (h) high-grade glioma spheroid cultured for a week and labeled with Hoechst (blue), Olig2 (yellow), and Phalloidine-488 (green) (glycerol-fructose clearing; 20x water objective). (D) Confocal images of human (h) rhabdomyosarcoma (top) and mouse $(\mathrm{m})$ neural crest cell (bottom) spheroids cultured for a week and labeled with Hoechst (blue), Actin (red), and Ki67 (green), respectively (glycerol-fructose clearing; 20x dry objective). (E) Confocal images of (h) 
high-grade glioma spheroid cultured for a week and labeled with Hoechst (blue) and Ki67 (green) (glycerol-fructose clearing; 40x water objective) (top left). Segmented images on the Hoechst channel and Ki67-positive (+) nuclear regions on the green channel were generated using high-content analysis software (see Supplementary Figure 1 and Table of materials) (bottom). Output given is the percentage of $\mathrm{Ki} 67^{+}$nuclei per segmented 3D structure (top right). Scale bar $=100 \mu \mathrm{m}$. Please click here to view a larger version of this figure.
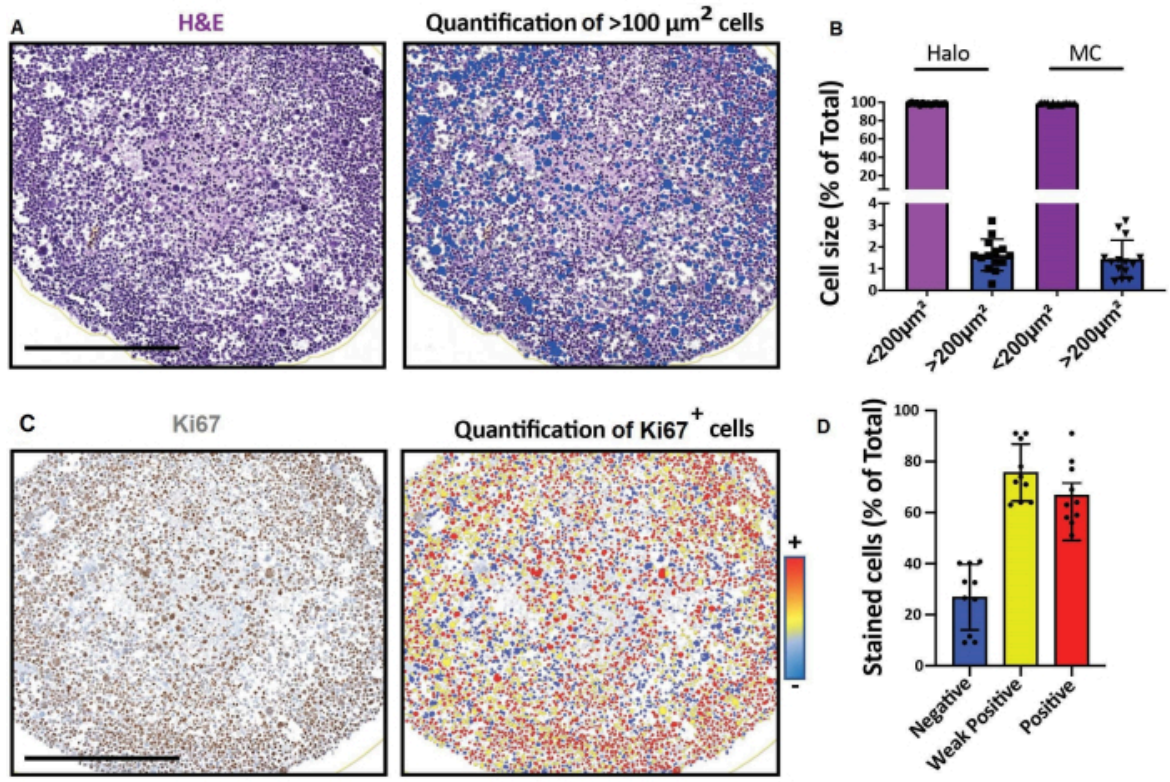

Figure 4: Representative results for imaging and analyses of 2D optical sections. (A, D) 2D section images of a 3D cell model (human rhabdomyosarcoma spheroids cultured for a month) obtained with a digital slide scanner and analyzed on a platform for fast digital quantitative analysis. (A) H\&E staining and detection of cells according to their size. Scale bar $=500$ $\mu \mathrm{m}$. (B) Histogram shows percentage of cells $<100 \mu \mathrm{m}^{2}$ and $>100 \mu \mathrm{m}^{2}$ detected using software for fast digital quantitative analysis (left: Halo) or manual counting (right: MC). (C) Ki67 staining and detection of cells according to the intensity of their 3,3'-diaminobenzidine (DAB) signal. Negative (blue), weakly positive (yellow), positive (red). Scale bar $=500 \mu \mathrm{m}$. (D) Histogram shows percentage of Ki67-negative, weakly positive, and positive cells. Abbreviations: H\&E = hematoxylin and eosin; $M C=$ manual counting. Please click here to view a larger version of this figure.

\section{Supplementary Figure 1: Overview of the steps in} the imaging analysis software. Analyses are based on the association of building blocks. Each building block corresponding to a function-segmentation, calculation, association, output definition-and offers multiple algorithms and variable selections to match the biological sample being imaged. The software provides multiple RMS (Ready Made Solution) analysis protocols that can easily be used and modified. Integrated image analysis protocols can be saved, applied to different datasets, and shared between users. Briefly, the analysis protocol implies sequential object segmentation: spheroid, nuclei and finally, Ki67 pockets 
(A488). Then, the mean intensity of the Ki67 pockets is calculated to further discriminate the positive events. Finally, nuclei encompassing Ki67 positive pockets are positively selected. Please click here to download this File.
Supplementary Figure 2: Overview of the procedure steps of the quantitative analysis software. Step

1. Upload the files using the Studies tab. Files will be opened in the Image Actions section. Step 2. Open the Annotations tab, then click on Layer Actions to design a new layer all around the structure using the circle tool of the toolbar. For non-circular structures, the pen tool can be used instead. Step 3. The toolbar can be used to design annotations and visualize the quantification with the $\sqrt{ }$ tool. Step 4. Open the Analysis tab, and select the best conditions for the analysis of the sample (several trials may be necessary here). Step 4.1. Use the Stain Selection section to set up the staining condition. In the event of several stains, these can be added and renamed, and the virtual color can be modified. The localization detection can be specified-nuclear or cytoplasm staining. Step 4.2. Use the Cell Detection section to set up the cell detection. This section will be the most important for the analysis. The Nuclear Contrast Threshold section will enable detection of all nuclei. Attention must be paid in case there are multiple population sizes, the software can detect several cells instead of a unique big one. Nuclear Size and Nuclear segmentation aggressiveness sections can be used to quantify cell size population ranges. Step 5. Description on how to run sample analysis. Follow steps shown in the figure. Annotation Layer section will run the setting only on this slide. The quantification can be visualized using the $\checkmark$ tool. Repeat steps $4.1-5$ until suitable quantification is achieved. Steps 6-6.1. These steps enable you to draw a figure using the software. Step 7. Quantification graphics obtained via software can be saved. Step 8. Data can be exported. Please click here to download this File. 


\section{Discussion}

Cell culture is an indispensable tool to uncover fundamental biological mechanisms involved in tissue and organ development, function, regeneration and disruption, and disease. Although monolayered 2D cell culture has predominated, recent research has shifted towards cultures generating $3 \mathrm{D}$ structures more reflective of in vivo cellular responses, owing notably to additional spatial organization and cell-cell contacts that influence gene expression and cellular behavior and could thus provide more predictive data $^{7}$. Nevertheless, many challenges remain, including the need for user-friendly staining and imaging techniques for detailed microscopic visualization and evaluation of complex 3D structures at the cellular and subcellular levels. In that context, detailed, robust, and complementary protocols have been provided to perform staining and cellular and subcellular resolution imaging of fixed in vitro 3D cell culture models ranging from $100 \mu \mathrm{m}$ to several millimeters in size.

This procedure presents two different strategies to deal with a large variety of sizes and types of in vitro 3D cell culture models. The choice of one (3D whole-mount analysis) or the other (2D sectioning analysis) will depend on the model used and issue investigated. 3D whole-mount analysis by confocal microscopy enables the visualization of cells with a field of depth of up to $200 \mu \mathrm{m}$, irrespective of the overall size of the $3 \mathrm{D}$ structure, whereas $2 \mathrm{D}$ sectioning is applicable to samples of any size, but visualization remains $2 \mathrm{D}$ dimensional. Below are some suggestions for troubleshooting and technical considerations.

Loss of 3D structures during the workflow is the most common drawback. They can remain adherent to the tips and tubes, which is why precoating tips and tubes with PBSBSA $0.1 \%$ solution is key. Moreover, it is crucial to let the $3 \mathrm{D}$ structures sediment between reagent changes and to perform all pipetting very carefully. As mentioned in the procedure, for all steps, if 3D structure sedimentation is too long, cells can be gently spun at $50 \times g$ for $5 \mathrm{~min}$ at RT. Depending on the aim of the study, the advantages/disadvantages of such a spinning step should be considered as centrifugation can compromise the shape of the 3D structures. Moreover, care should be taken to preserve this morphology during the fixation step because cystic organoids tend to collapse. Fixing structures under $400 \mu \mathrm{m}$ in size should prevent structural changes.

For optimal immunolabeling, recovery of organoids from their $3 D$ matrices is a crucial step. The $3 D$ matrix can impede adequate antibody penetration or lead to high background staining because of non-specific binding to the matrix. ECM removal may alter the morphology of the outer segments of organoids (notably in case of small cellular protrusions extending from studied 3D structures) and partially hamper analyses. For such 3D structures, the matrix can be retained throughout the procedure; however, culture conditions should be carefully adapted to grow cells in a minimum amount of matrix to prevent insufficient penetration of solutions and antibodies and to avoid successive washing steps aimed at reducing excessive background noise $e^{6,8}$.

The optical clearing step described in this protocol in the 3D whole mount staining section is pertinent for the imaging of $3 \mathrm{D}$ structures up to $150-200 \mu \mathrm{m}$ in depth instead of 50-80 $\mu \mathrm{m}$ without clearing. Compared to other clearing methodologies that often requiring several weeks and using toxic clearing agents, a previously published fast and safe clearing step was used in this protocol ${ }^{4,9}$. In addition, this clearing step is reversible, and new antibodies can be added to the initial staining with no loss of resolution or brightness ${ }^{4}$. Nevertheless, depending on the $3 \mathrm{D}$ cell 
culture model studied, a depth of $150-200 \mu \mathrm{m}$ might not be sufficient to image the 3D structure in an informative way, and this clearing protocol can cause changes in the general morphology of spherical, monolayered organoids with large lumens ${ }^{4}$. Users should carefully design their experiment, and if necessary, optimize the timing of the permeabilization/ blocking step (to allow penetration of antibodies and solution), the clearing step (to penetrate deeper than $200 \mu \mathrm{m}$, specimens should be totally cleared), and image acquisition. The two most prevalent technologies available in core facilities would be light sheet and confocal microscopy. Users will need to carefully choose a technology based on the size of their $3 \mathrm{D}$ structures and their biological question ${ }^{10}$. However, compared to confocal microscopy, light sheet microscopy resolution obtained for such deep structures remains suboptimal for obtaining subcellular resolution.

Here, a detailed and robust process has been reported that is dedicated to paraffin embedding of single samples. Interestingly, Gabriel et al. recently developed a protocol embedding 3D cell cultures in paraffin with an increased throughput. They used a polydimethylsiloxane (PDMS) mold to confine $963 \mathrm{D}$ structures in a microarray pattern in one block, providing novel perspectives for studies on 3D tumor models encompassing more groups, time points, treatment conditions, and replicates ${ }^{11}$. However, this method requires extensive skills and machinery, notably for the fabrication of the premold used to created PDMS molds.

In summary, this paper describes two different, complementary, and adaptable approaches enabling the acquisition of accurate and quantitative information on architectural and cellular composition of 3D cellular models. Both parameters are crucial for studying biological processes such as intratumoral cellular heterogeneity and its role in resistance to treatments.

\section{Disclosures}

The authors have nothing to disclose.

\section{Acknowledgments}

This work was supported by the St Baldrick's Robert J. Arceci Innovation Award \#604303.

\section{References}

1. Ryu, N. E., Lee, S. H., Park, H. Spheroid culture system methods and applications for mesenchymal stem cells. Cells. 8 (12), 1-13 (2019).

2. Bartfeld, S., Clevers, H. Stem cell-derived organoids and their application for medical research and patient treatment. Journal of Molecular Medicine. 95 (7), 729-738 (2017).

3. Cui, X., Hartanto, Y., Zhang H. Advances in multicellular spheroids formation. Journal of the Royal Society, Interface. 14 (127) (2017).

4. Dekkers, J. F. et al. High-resolution 3D imaging of fixed and cleared organoids. Nature Protocols. 14, 1756-1771 (2019).

5. Broutier, L. et al. Culture and establishment of selfrenewing human and mouse adult liver and pancreas 3D organoids and their genetic manipulation. Nature Protocols. 11 (9), 1724-1743 (2016).

6. Rezanejad, H., Lock, J. H., Sullivan, B. A., BonnerWeir, S. Generation of pancreatic ductal organoids and whole-mount immunostaining of intact organoids. Current Protocols in Cell Biology. 83 (1), ee82 (2019). 
7. Edmondson, R., Broglie, J. J., Adcock, A. F., Yang, L. Three-dimensional cell culture systems and their applications in drug discovery and cell-based biosensors. Assay and Drug Development Technologies. 12 (4), 207-218 (2014).

8. McCray, T., Richards, Z., Marsili, J., Prins, G. S., Nonn, L. Handling and assessment of human primary prostate organoid culture. Journal of Visualized Experiments: JoVE. (143), 10.3791/59051 (2019).

9. Ueda, H. R. et al. Tissue clearing and its applications in neuroscience. Nature Reviews: Neuroscience. 21 (2), $61-79(2020)$.

10. Lazzari, G. et al. Light sheet fluorescence microscopy versus confocal microscopy: in quest of a suitable tool to assess drug and nanomedicine penetration into multicellular tumor spheroids. European Journal of Pharmaceutics and Biopharmaceutics. 142, 195-203 (2019).

11. Gabriel, J., Brennan, D., Elisseeff, J. H., Beachley, V. Microarray embedding/sectioning for parallel analysis of 3D cell spheroids. Scientific Reports. 9, 16287 (2019). 